\title{
Une proposition d'analyse culturelle des gwerzioù
} Comportements et sensibilités dans la Basse-Bretagne d'Ancien Régime d'après les complaintes en langue bretonne

\section{Éva Guillorel}

\section{Q OpenEdition}

1 Journals

Édition électronique

URL : http://journals.openedition.org/abpo/895

DOI : $10.4000 / a b p o .895$

ISBN : 978-2-7535-1501-7

ISSN : 2108-6443

Éditeur

Presses universitaires de Rennes

Édition imprimée

Date de publication : 30 mars 2006

Pagination : 25-52

ISBN : 978-2-7535-0273-4

ISSN : 0399-0826

\section{Référence électronique}

Éva Guillorel, « Une proposition d'analyse culturelle des gwerzioù », Annales de Bretagne et des Pays de I'Ouest [En ligne], 113-1 | 2006, mis en ligne le 30 mars 2008, consulté le 30 avril 2019. URL : http:// journals.openedition.org/abpo/895; DOI : 10.4000/abpo.895 


\title{
Une proposition d'analyse culturelle des gwerzioù
}

\author{
Comportements et sensibilités \\ dans la Basse-Bretagne d'Ancien Régime \\ d'après les complaintes en langue bretonne
}

\author{
Eva GUILLOREL \\ Agrégée d'histoire, \\ CRHISCO - université Rennes 2 Haute-Bretagne
}

Le regard porté sur les complaintes en langue bretonne - ou gwerzioù par les historiens depuis le début des grandes collectes de chansons du $\mathrm{XIX}^{\mathrm{e}}$ siècle est le reflet des évolutions des préoccupations de la discipline historique : suite aux premières études érudites de quelques chants remarquables mis en relation avec une histoire événementielle telle qu'elle pouvait se faire à la fin du XIX ${ }^{\mathrm{e}}$ siècle, l'approche s'est largement diversifiée en lien avec l'essor de l'histoire des mentalités et elle s'est enrichie des apports d'autres disciplines, notamment de l'ethnologie. Les problèmes méthodologiques spécifiques à l'étude de cette littérature orale ont fait l'objet de plusieurs analyses au cours de ces dernières décennies, qui ont abouti à la conviction d'une nécessaire confrontation entre sources orales et écrites ${ }^{1}$. Toutefois, l'originalité que présentent les complaintes en langue bretonne en tant que sources orales populaires ${ }^{2}$ et quasi directes explique l'intérêt qu'elles ne cessent de susciter ${ }^{3}$.

1. Le récent article publié par Michel Nassiet fait le point sur ces questions de critique méthodologique. NASSIET, Michel, " La littérature bretonne et l'histoire ", Annales de Bretagne et des Pays de l'Ouest, tome 106, n 3, 1999, p. 35-64.

2. Le terme fait ici référence au milieu dans lequel les chansons ont été recueillies, à défaut de pouvoir s'avancer avec certitude sur la question problématique de l'auteur de ces compositions. L'expression " chansons populaires ", malgré les ambiguïtés qu'elle induit, a néanmoins été préférée à celle de " chansons traditionnelles " empruntée au vocabulaire des ethnologues : cette dernière présente en effet l'inconvénient de faire parfois référence, à tort, à un concept de " société traditionnelle " à la connotation figée qui apparaît difficilement recevable dans le cadre d'une analyse historique.

3. Cet article pose une part importante des orientations de recherche mises en œuvre dans la préparation d'une thèse d'histoire moderne à l'université Rennes 2, réalisée avec le soutien financier de la région Bretagne. 
En l'absence de critères élémentaires permettant une critique de la source envisagée - date, auteur, contexte -, la première et indispensable étape dans l'analyse historique de ces chansons a consisté pour les folkloristes, les historiens et plus largement les érudits qui se sont passionnés pour cette question depuis les années 1820, à mettre en évidence le lien entre une chanson particulièrement détaillée et un événement attesté par d'autres sources écrites. Il a pu être ainsi constaté à de nombreuses reprises que des complaintes transmises oralement le plus souvent sur plusieurs siècles pouvaient avoir conservé, parfois avec un degré de fiabilité remarquable, le souvenir d'événements précis. Une trentaine de gwerzioù antérieures à la période révolutionnaire ont ainsi pu être finement datées, notamment entre les dernières décennies du XVI ${ }^{\mathrm{e}}$ siècle et la première moitié $\mathrm{du} \mathrm{XVIII}^{\mathrm{e}}$ siècle $^{4}$ : c'est à la fois beaucoup et peu en comparaison de la masse foisonnante que constituent les chansons collectées tant par écrit que par oral - par le biais d'enregistrements sonores -, qui peuvent être évaluées en milliers de pièces comprenant parfois de nombreuses variantes d'un même chant.

C'est toutefois sous un angle différent que l'étude des gwerzioù est ici envisagée, non plus à partir d'une approche événementielle, dont la pertinence mais également les limites sont manifestes, mais dans l'optique d'une analyse culturelle des chansons. En partant du postulat que les complaintes en langue bretonne peuvent, en y portant un regard critique vigilant, s'avérer des sources fiables dans le cadre d'une étude en histoire moderne, la voie est ouverte à une réflexion dont l'enjeu est tout autre : à partir d'éléments d'ordre non événementiel - manières d'être et de penser, comportements, rapport aux autres et regard sur les autres, éléments de culture matérielle -, il s'agit de tenter d'esquisser une datation culturelle de ces gwerzioù par le biais de rapprochements avec d'autres sources écrites, dans une approche qui élargit ainsi considérablement les perspectives d'analyse historique.

\section{À la recherche d'une datation culturelle des complaintes en langue bretonne}

\section{Les difficultés liées à l'incertitude entourant la date de composition des gwerzioù}

Face à l'incertitude chronologique qui caractérise les complaintes en langue bretonne, une approche semble devoir être retenue : partir de la gwerz telle qu'elle est attestée par écrit au XIX ${ }^{\mathrm{e}}$ siècle, seule certitude d'un état de la chanson à un moment donné, et tenter de remonter en amont, afin de cerner dans quelle mesure la chanson est révélatrice de sensibilités ou de gestes plus anciens, dont l'expression se rapporterait à la période de création du canevas premier de la chanson.

4. Dans son article déjà cité, Michel Nassiet présente la synthèse des principales études de cas ayant permis la datation précise de complaintes, dont il propose une liste. 
Plusieurs cas se présentent alors. La chanson peut tout d'abord fournir des données attestées de manière ancienne (par exemple, la pratique d'enterrement massif à l'intérieur de l'église), que l'on ne retrouve plus au $\mathrm{XIX}^{\mathrm{e}}$ siècle à l'époque de la collecte du chant. Il est alors possible de définir chronologiquement la période au cours de laquelle ce fait est attesté ou, le plus souvent, la période à partir de laquelle il n'est plus attesté.

Dans un second cas, au contraire, la gwerz comporte un élément que l'on sait plus tardif, par exemple l'emploi d'un vocabulaire républicain (avec des références à l'assemblée nationale ou aux soldats de la nation). Si la chanson est effectivement de conception récente, des indices permettent la plupart du temps de le repérer : un style différent se rapprochant de celui qui s'épanouit nettement au XIX ${ }^{\mathrm{e}}$ siècle par le biais des feuilles volantes, et souvent - mais pas toujours - la faible diffusion spatiale du chant et le nombre restreint de variantes ${ }^{5}$. Mais la gwerz peut être également de conception ancienne, l'élément en question ayant été introduit ultérieurement, suivant le processus habituel d'évolution du chant, dans le but de l'actualiser. La difficulté est alors de faire la part, dans la même chanson, entre des éléments anciens et des éléments interpolés - insérés ultérieurement - qui peuvent surgir à tout moment ${ }^{6}$. Il semblerait logique en effet que, dans une société qui évolue nécessairement, la chanson transmise fréquemment sur plusieurs siècles soit affectée par des réactualisations; leur mise en évidence, notamment par le relevé d'anachronismes et par la comparaison entre différentes variantes d'un même chant, permet de discerner une évolution des sensibilités : les interpolations tardives sont ainsi le signe qu'un élément n'est plus crédible, dorénavant incompris pour le chanteur et l'auditoire, qu'il a perdu sa force d'évocation, et est donc supprimé ou remplacé par un autre plus proche d'une sensibilité nouvelle. Ces transformations restent toutefois minoritaires, et Alain Croix remarque à ce sujet que « la mémoire populaire en effet - qui le nierait - vit, est donc sensible, même avec retard, aux évolutions de tous ordres, mais en même temps apparaît comme un conservatoire exceptionnel. [...] Le texte par consé-

5. Ces critères doivent être considérés avec prudence : Donatien Laurent, prenant le cas de chants du Barzaz Breiz (recueil publié pour la première fois en 1839 par Théodore Hersart de La Villemarqué) considérés jusque-là comme douteux, comme Merlin-Barde ou Le Faucon, a cependant montré que certaines complaintes peuvent n'avoir été collectées qu'une fois, et portent néanmoins toutes les marques de chants transmis sur la longue durée. Laurent, Donatien, Aux sources du Barzaz-Breiz. La mémoire d'un peuple, Douarnenez, 1989, p. 286-312. Par ailleurs, Daniel Giraudon, dans son étude sur la complainte concernant l'assassinat de la Dame de Kerizel en 1663, a montré que cette gwerz, encore bien connue localement plus de trois siècles plus tard, ne s'était pourtant pas diffusée en dehors de la commune où a eu lieu le fait divers. GIRAUDON, Daniel, "Itron a Gerizel ", Bulletin de la Société d'Émulation des Côtes-du-Nord, 1984, p. 60-77.

6. L'analyse a été menée par Alain Croix au sujet de la gwerz de Skolan (par ailleurs mise en relation par Donatien Laurent avec un poème gallois du XII ${ }^{\mathrm{e}}$ siècle) dans laquelle il relève des anachronismes en matière de sensibilité religieuse, à savoir des allusions au purgatoire et à l'utilisation d'un chapelet. CROIX, Alain, La Bretagne aux XVI $-X V I I^{e}$ siècles. La vie, la mort, la foi, Paris, Maloine, 1981, t. 2, p. 930-931. 
quent témoigne beaucoup plus de l'époque de ses origines que de celle où le transcripteur le relève ${ }^{7}$ ".

Dans un dernier cas, de loin le plus fréquent, la chanson présente des traits valables sous l'Ancien Régime, mais également au XIX ${ }^{\mathrm{e}}$ siècle. En l'absence de tout élément permettant d'envisager une datation plus précise, nous sommes ici réduits à la formulation de suppositions : il est vraisemblable - plus ou moins selon les cas - que des données rencontrées dans un chant collecté au XIX ${ }^{\mathrm{e}}$ siècle se rapportent à un contexte d'Ancien Régime, dans la mesure où l'on retrouve ces mêmes données dans la documentation écrite relative à cette époque. L'appréciation de ce critère doit alors se faire au cas par cas.

Plusieurs angles d'analyse sont ainsi proposés. Cette nécessaire multiplicité de l'approche tient à la volonté de limiter un risque omniprésent : celui de systématiquement placer tous les chants sur le même plan, de les comparer comme s'ils provenaient d'un même lieu et d'une même époque, et ainsi de s'enfermer dans la vision d'une société traditionnelle figée, d'une communauté de culture qui n'aurait pas évolué entre la création de ces chansons et la date de leur collecte.

\section{Typologie de la vraisemblance, sphères de la crédibilité : la validité du chant en soi}

L'incertitude chronologique caractérisant la chanson de transmission orale amène toute analyse culturelle de ces complaintes à ne jamais se situer dans le domaine de la "vérité ", mais toujours dans celui du vraisemblable, envisagé selon deux aspects très différents : tout d'abord, comment est appréhendée la question de la vraisemblance du chant par le chanteur et l'auditeur eux-mêmes, acteurs vivants de cette littérature orale et de sa transmission dans son cadre d'expression habituel ${ }^{8}$ ? Comment, ensuite, est abordée cette même question par l'historien, extérieur à ce cadre, ayant fait de la chanson une source d'étude, et l'ayant par là même extraite de son contexte premier? Ce sont deux regards radicalement différents posés sur le même support.

Pour le chanteur et l'auditoire, la vraisemblance est une donnée essentielle dans le cas des gwerzioù, donnée qui entre dans la définition même de ce type de chants. Toute la crédibilité de la complainte, donc sa raison d'être, repose sur la conviction que l'histoire racontée est vraie. Cette affirmation est facilitée par le fait que la chanson est largement transmise dans le cadre de la famille, dans une société où la parole des anciens est valorisée. Ce rapport particulier à la gwerz avait déjà été souligné par le collecteur La Villemarqué en 1845 : "Quand un paysan breton veut louer une

7. Ibidem, t. 2, p. 927-930.

8. Cette question n'a véritablement de sens que dans le contexte d'une société rurale ancienne qui offre de réelles conditions de diffusion et de transmission du répertoire chanté de transmission orale, c'est-à-dire essentiellement avant les années 1950. 
œuvre de ce genre, il ne dit pas : “c'est beau!", il dit : “c'est vrai ${ }^{9}$ !" " Ce à quoi Donatien Laurent ajoute : "On comprend dès lors l'importance de ce type de chansons, censées porter la Vérité du groupe, dans une société orale où la parole a un prix très supérieur à celui qu'elle a dans nos civilisations de l'écrit. On comprend la responsabilité de l'auteur de la chanson - qui doit n'y mettre que ce qu'il sait être "vrai" - comme celle du transmetteur qui, sous le contrôle de la communauté, doit rester fidèle à cette "vérité ${ }^{10 "}$ " " Bien entendu, entre la conviction de véracité et la véracité effective, la distance peut être grande. Il n'en ressort pas moins que l'importance accordée à ce critère limite fortement les possibilités d'évolution de la gwerz: y apporter des modifications volontaires impliquerait de s'éloigner de la vérité du chant, et donc de perdre en crédibilité. En cela, un fossé énorme sépare les gwerzioù des chansons plus légères - les sonioù -, qui n'ont pas pour objectif de " dire vrai ".

Pour l'historien, la question de la vraisemblance de la complainte se pose en d'autres termes : d'une part, jusqu'à quel point la volonté du chanteur et de l'auditoire de " dire vrai " a pour conséquence de conserver la structure première de la chanson, et donc d'être révélatrice de l'époque à laquelle la gwerz a été composée? Quels éléments du chant se sont transmis le plus fidèlement, et à quels critères obéissent les mécanismes d'évolution - donc de transformation - du récit? Est-il vraisemblable que des informations transmises uniquement par le biais de la mémoire soient conservées sans altération sur plusieurs siècles? Les nuances du raisonnement se formulent alors en degrés de vraisemblance : très vraisemblable, l'élément sera retenu; jusqu'au point où le degré de vraisemblance semblera trop faible - avec toute la subjectivité qu'implique ce jugement - et où l'élément sera écarté de l'analyse.

\section{La chanson, reflet des sensibilités}

Les oublis, transformations, interpolations qui caractérisent l'évolution d'un chant sont révélateurs de la sensibilité du chanteur et de son auditoire. La complainte propose ainsi une relecture de faits et d'attitudes selon les inspirations et les aspirations des personnes qui créent ${ }^{11}$ et des groupes qui véhiculent ce support. Ce second cas nous est pour ainsi dire seul connu, par le biais des chanteurs auprès desquels ont été recueillies les complaintes : il s'agit pour l'essentiel de domestiques, mendiants et représentants des petits métiers artisanaux, qui gravitent souvent dans l'environnement immédiat des collecteurs du XIX ${ }^{\mathrm{e}}$ siècle ${ }^{12}$. Ces derniers ont de

9. Préface à la seconde édition du Barzaz Breiz, cité dans : LAURENT, Donatien, « Histoire et poésie chantée : l'exemple de la Bretagne ", Historiens et Géographes, n 318, mars-avril 1998, p. 111.

10. Ibidem.

11. Se pose là encore le problème d'identification de l'auteur.

12. Luzel a ainsi recueilli la presque totalité des quatre volumes de chants qu'il a publiés dans le seul canton de Plouaret. 
plus effectué leur propre tri parmi les chanteurs, en fonction de critères qui ne sont pas toujours conscients et qui ont trait à leurs opportunités de collectage ou à leurs convictions politiques et religieuses. Les chanteurs eux-mêmes choisissent leur répertoire en fonction de leur sensibilité personnelle et de l'auditoire qui leur fait face, et ne chantent vraisemblablement pas toujours les mêmes pièces dans une veillée ou en présence d'un collecteur lettré et extérieur à la communauté villageoise.

C'est en définitive une vision très ciblée du monde qui transparaît à travers le chant, un univers mis en scène dont la description nous est parvenue par l'intermédiaire d'un éventail de chanteurs bien particuliers, issus d'une société rurale et bretonnante, qui transposent dans la chanson la sensibilité de leur milieu. À titre d'exemple, le jugement porté sur le monde mal connu de la ville, d'ailleurs peu présent dans les chansons issues des grandes collectes du XIX ${ }^{\mathrm{e}}$ siècle, est bien celui - particulièrement original par rapport aux sources écrites - d'une communauté qui lui est largement extérieure et qui regarde l'univers urbain et ses bourgeois d'un œil peu favorable ${ }^{13}$.

De plus, cette société se met en scène dans des complaintes qui obéissent à des canons précis. La comparaison entre l'utilisation du décor de l'auberge dans les gwerzioù et dans les sonioù - qui forment un répertoire souvent plus enjoué de chants d'amour ou de métiers véhiculés par les mêmes chanteurs - est à cet égard révélatrice. Dans les complaintes, l'auberge est peu mentionnée, et rarement représentée comme un lieu de fête et de débordements. Elle sert de cadre impersonnel aux habituels récits de crimes, qui se situent d'ailleurs la plupart du temps sur le chemin de l'auberge et non dans le lieu même ${ }^{14}$. On rencontre tout de même quelques rares descriptions de comportements festifs dans les tavernes, mais ceuxci y sont toujours condamnés et s'inscrivent dans un discours fortement teinté de morale religieuse ${ }^{15}$. Dans les sonioù au contraire, la taverne et l'au-

13. On peut ainsi noter l'évocation, dans une complainte recueillie par La Villemarqué, du diable "gwisket evel ar bourchis ", c'est-à-dire " habillé comme un bourgeois ", dans une description révélatrice du peu d'estime accordée à l'un comme à l'autre. LAURENT, D., 1989, op. cit., p. 73-75.

14. Sur plus de 850 chansons ici retenues, 35 mentionnent une auberge et constituent les versions correspondant à treize chants-types. Parmi ces derniers, cinq appartiennent au répertoire des sonioù. Trois autres, se rapportant cette fois au répertoire des gwerzioù, évoquent l'auberge à titre vraiment anecdotique et sans réel intérêt. Il n'existe en fait qu'un seul chant-type où un meurtre a lieu à l'intérieur de l'auberge (à savoir la gwerz sur le petit marchand de Rouen) et deux où un crime a lieu sur le chemin du retour de l'auberge (celles du clerc Guégan et de Perrine Le Mignon), auxquels on peut encore ajouter deux derniers chants-types où l'auberge est effectivement un lieu de débordements et de violences verbales.

15. C'est ainsi que saint Gwenaël refuse de secourir des naufragés malgré les prières qui lui sont adressées, en prétextant qu'ils ne font que boire et jouer aux cartes à l'auberge au lieu d'aller à la grand-messe et aux vêpres. HERRIEu, Loeiz, Guerzenneu ha Soñnenneu Bro-Guéned. Chansons populaires du pays de Vannes, Lorient, 1997 (1 $1^{\text {re }}$ éd. 1911), p. 159-160. 
berge sont bien présentes et remplissent le rôle plus conventionnel de lieu de divertissement et de plaisirs. Le ressort tragique développé dans les gwerzioù semble donc exclure toute représentation de conduites badines ou répréhensibles dans un lieu qui fait pourtant partie du quotidien. Le récit reste dans un ton grave et décrit un univers austère dans lequel les comportements de chacun sont régis selon une morale de bienséance et jugés en fonction de leur convenance à cette morale.

Les jugements de valeur explicites ou implicites donnent à voir dans les complaintes une conception morale de la société, où les fautifs doivent être punis et où les innocents injustement frappés sont considérés avec compassion. La société mise en scène prend la forme d'une communauté où chacun doit rester à sa place et maintenir son rang et sa respectabilité et, si les débordements occupent une large place, ils sont suivis de justes représailles. Il n'est pas inutile de rappeler la dualité du terme gwir en breton, dont les récits de gwerzioù vérifient parfaitement le sens; Donatien Laurent écrit ainsi que " gwir, en son sens propre, qualifie tant ce qui est véridique, authentique, objet de croyance, que ce qui est bon, droit, juste, équitable; la justice, le droit se disent ici du même mot que la vérité : ar gwir ${ }^{16}$ ". La prise en considération de cette notion essentielle est la porte ouverte à la compréhension de la morale des complaintes et du regard sur la justice qui y est porté : le jugement doit redresser et punir les déviances. Ainsi se comprend le regard porté sur les puissants et l'image ambivalente qu'occupe le noble, à la fois suborneur et meurtrier mais également défenseur des opprimés et protecteur des pauvres, dans des complaintes au net parti pris dont le jugement moral dépasse une simple confrontation entre groupes sociaux. Quand la justice des hommes fait défaut, la gwerz fait intervenir d'autres procédés pour rétablir l'ordre exigé par les conventions du genre : miracles, édification des victimes de destinées tragiques au rang de martyres ou encore modification pure et simple d'éléments non conformes aux attentes de l'auditoire; c'est ainsi que la relecture de la célèbre exécution de François de Montmorency-Bouteville et de son cousin le comte Des Chapelles en 1627, condamnés à mort à titre exemplaire pour avoir bravé l'interdit royal et s'être battus en duel, aboutit dans la complainte à une totale transformation de la réalité en évoquant une grâce fictionnelle accordée par le roi au comte cornouaillais, tandis que Montmorency n'est pas même évoqué dans la chanson ${ }^{17}$.

On se situe donc bien dans le domaine d'une histoire des sensibilités : l'intérêt de la chanson populaire en tant que source ne réside pas dans la mise en évidence de "vérités ", mais bien plus dans l'analyse de la perception de ce qui est considéré comme vrai. Le champ d'étude n'est pas celui d'une histoire de la criminalité ou d'une histoire des rapports sociaux

16. LAURENT, D., « La gwerz de Skolan et la légende de Merlin », Ethnologie française, 1971, 3-4, p. 29.

17. BllLACoIs, François, Le Duel dans la société française des XVI'-XVIT siècles. Essai de psychosociologie historique, Paris, 1986, p. 270-273. 
que pourraient révéler les gwerzioù, mais celui du regard porté sur certaines réalités et de la représentation que la société se fait d'elle-même, avec ses codes, ses normes et sa morale.

\section{De larges possibilités d'exploitation}

Cette méthodologie mise en place, il s'agit désormais de mesurer l'intérêt et la richesse que constitue la matière des gwerzioù dans le domaine d'une analyse en histoire culturelle. Une double approche peut être privilégiée, selon le type de données apportées par la chanson. Concernant une première catégorie d'éléments, il s'agit d'apprécier si leur confrontation avec la documentation écrite permet de les replacer dans un contexte chronologique défini, et d'analyser leur pertinence dans un tel cadre. Concernant une deuxième catégorie d'éléments attestés cette fois sur le long terme sans rupture chronologique, valables - ou tout au moins crédibles - à l'époque de la collecte, on peut s'interroger sur la possibilité d'une mise en évidence de traits de culture propres à la Bretagne bretonnante, et juger la valeur de l'apport particulier de la chanson dans un domaine déjà largement documenté par des sources écrites.

Toujours sous-tendues par la volonté de raconter une histoire vraie, les complaintes regorgent de mentions précises concernant des domaines aussi variés que la vision portée sur les hiérarchies sociales, les normes langagières ou vestimentaires, le rapport à l'écrit ou les comportements religieux, la liste ainsi proposée étant loin d'être exhaustive. Qu'il s'agisse de la description d'une pièce de vêtement révélant un rang social ou d'un geste traduisant le respect ou le mépris, la nécessaire concision exigée par la structure versifiée donne un relief particulier à chacune des mentions retenues, qui ne sont par là même jamais dépourvues de sens : le chanteur se voit contraint de ne mentionner qu'un détail significatif, qui prend d'autant plus de valeur que la brièveté du récit chanté le rend souvent rare.

\section{Un exemple de l'apport des complaintes dans le domaine de la culture matérielle : les mentions vestimentaires}

Le vêtement, marqueur le plus visible de l'appartenance à un groupe et à une époque, est évidemment très présent dans la chanson. Il est avant tout un signe extérieur de richesse dont la qualité, la profusion ou l'absence révèlent le rang et dont la compréhension passe par la connaissance de codes sociaux. La description d'un pauvre venu demander asile est à cet égard évocatrice : "Nen devoa na tog na bonnet na rochet dindana ${ }^{18}$ "; en un vers, l'identité sociale est donnée sans équivoque. Au contraire, Marguerite Charlès exprime sa richesse par la description de ses coiffes :

18. «Il n'avait ni chapeau ni bonnet ni chemise de dessous ». Collection Penguern, manuscrit 95 , folio 315 , Bibliothèque nationale de France. 


\section{"Tric'houec'h dousenn 'zo anezho./Tric'houec'h en lienn Hollanda, Ha kement-all en batista ${ }^{19}$."}

Le vêtement apparaît comme un indice chronologique intéressant : les fréquentes mentions de la toile de Hollande renvoient au contexte économique du $\mathrm{XVII}^{\mathrm{e}}$ siècle et à la concurrence étrangère sur le marché des crées, les toiles léonardes; celles concernant Quintin sont plus révélatrices de l'essor des toiles bretagnes au XVIII ${ }^{\mathrm{e}}$ siècle. Les nombreuses mentions de " tok kastor ${ }^{20}$ " ne se comprennent que replacées dans le contexte du règne de Louis XIII, où ce grand feutre gris empanaché d'une longue plume d'autruche est la coiffure caractéristique de la cour. La profusion de dentelles est quant à elle significative d'une époque plus tardive et voit son essor dans les milieux aristocratiques sous le règne de Louis XIV.

La mention d'une pièce de vêtement caractéristique d'une époque donnée n'est néanmoins pas à elle seule un argument de poids en faveur de la datation de l'époque de composition d'un chant. Dans ce domaine plus que dans tout autre, les modifications - il serait plus juste de parler de renouvellements - sont courantes, puisque le costume, par définition, se démode vite, notamment dans les milieux aisés, et est un élément extérieur visible qui interpelle : son actualité participe à la création d'un cadre significatif pour le chanteur et l'auditoire. La transformation des données vestimentaires permet donc une rénovation facile et efficace du chant. Sur ce terrain, certaines interpolations apparaissent de manière évidente. La ballade exceptionnellement longue autour du personnage de Merlin, recueillie par La Villemarqué, dont l'authenticité et l'ancienneté sont défendues par Donatien Laurent qui considère cette pièce comme " un unique et ultime surgeon d'un fragment d'un cycle médiéval armoricain ${ }^{21}$ ", comporte parallèlement à de nombreux archaïsmes tant dans son lexique que dans sa forme métrique une mention anachronique : cent robes de laine blanche "dantelleset bed ar pen ${ }^{22}$ " sont portées par les prêtres de la noce, constituant un ajout de toute évidence plus tardif ${ }^{23}$.

On peut toutefois, lorsqu'un chant comporte plusieurs indices chronologiques cohérents et repérables dans les différentes versions, envisager une datation présumée pour sa composition. C'est par exemple le cas d'une gwer $z^{24}$ dans laquelle la mention conjuguée d'habits passementés - le mot est attesté dès le milieu du XvI ${ }^{\mathrm{e}}$ siècle -, d'un castor servant de couvre-chef

19. " J'en ai dix-huit douzaines;/Dix-huit douzaines en toile de Hollande,/Et autant en batiste ". LuZEL, François-Marie, Chants et chansons populaires de la Basse-Bretagne. Gwerziou II, Paris, 1971, réimpression de l'édition de 1874, p. 74-79. Il faut toutefois tenir compte, dans ces descriptions du luxe des puissants, de la capacité d'exagération qui caractérise la littérature orale.

20. "Chapeau castor".

21. LAURENT, D., 1989, op. cit., p. 287-296.

22. "Garnies de dentelle jusqu'au bout".

23. La dentelle fait son apparition $\mathrm{au} \mathrm{XVI}^{\mathrm{e}}$ siècle simultanément en Italie et en Flandres. DelPIERRE, Madeleine, Se vêtir au XVIII siècle, Paris, 1996, p. 66-67.

24. La jeune fille et les trois capitaines. Collection Penguern, manuscrit 111, folio 147. 
et du terme militaire de cadet, qui se diffuse au XVII ${ }^{\mathrm{e}}$ siècle, donne entre autres à penser que le chant a été composé au XVII ${ }^{\mathrm{e}}$ siècle. Un argument supplémentaire joue en faveur de cette hypothèse : la correspondance entre cette pièce et la chanson en langue française La fille qui fait la morte pour son honneur garder a été établie et l'étude de Georges Doncieux au sujet de ce chant, basée sur un travail lexical et syntaxique, aboutit à la même conclusion concernant l'époque de composition de la pièce en français ${ }^{25}$.

\section{La richesse des complaintes dans le domaine des comportements religieux}

Dans le cadre d'une étude de la sensibilité religieuse, les complaintes apparaissent comme une documentation particulièrement prometteuse et propre à enrichir les analyses déjà solides ayant mis en évidence les spécificités des comportements en Basse-Bretagne, dans la mesure où les gwerzioù sont porteuses d'attitudes insérées dans le contexte qui est le leur et qui émanent du milieu même qui entretient ces pratiques : on touche ici une strate particulièrement profonde et enracinée de la culture populaire rurale. Strate intime parfois, qui se révèle par des paroles ou des gestes individuels, elle prend aussi la forme d'expressions collectives où se retrouve la communauté autour de comportements fédérateurs.

Les demandes d'intercessions, la rédaction de testaments ou la description d'enterrements sont des sujets graves, pour lesquels on peut supposer que la mise en chanson aura recherché, peut-être encore plus que pour d'autres sujets, à se rapprocher d'une sensibilité vécue et partagée par la communauté rurale. Les grands bouleversements initiés par le clergé formé aux idées posttridentines sont une réalité dont il faut toutefois bien tenir compte : la sensibilité, les croyances et les gestes ont changé, sur certains aspects plus vite et plus profondément que sur d'autres, au cours des quelques siècles durant lesquels ont été composés et transmis les chants en langue bretonne ici étudiés. La connaissance fine de l'évolution chronologique en ce domaine peut constituer un formidable atout dans l'étude des gwerzioù en permettant, lorsque sont cernées les éventuelles interpolations plus tardives, de dater le chant.

À travers certains gestes, ce sont des particularités propres à la BasseBretagne que l'on peut saisir. Les demandes d'intercession aux saints révèlent par exemple la diversité des noms de saints spécialistes invoqués, reflet de la richesse culturelle de la Bretagne bretonnante à cet égard : c'est ainsi qu'une version de la gwerz sur la mort en duel de Des Tourelles, se rapportant à un événement daté en 1624, relate que le mourant demande une messe à Saint Iboan pour le soulager de ses souffrances ${ }^{26}$. Les com-

25. DoncieuX, Georges, "La fille qui fait la morte pour son honneur garder ", Mélusine, t. $6,1892-1893$, p. 145-151.

26. Collection Penguern, manuscrit 90, folios 63-65. À rapprocher de saint Diboan, dont le nom est une traduction littérale de la fonction, puisque diboan signifie "sans douleur " en breton. 
plaintes présentent un ensemble particulièrement fourni de prières et de promesses de dons qui ont l'avantage unique, par rapport à d'autres sources écrites, de nous livrer sans intermédiaire, dans leur contexte immédiat et à la forme directe, la formulation de vœux parfois très détaillés qui correspondent aux demandes attestées par ailleurs : objets liturgiques, exvoto en forme de bateau ou dons d'une ceinture de cire faisant le tour de l'église. L'accomplissement des promesses faites, suite à l'obtention d'un miracle, trouve là encore une large place dans les complaintes, notamment à travers la réalisation de pèlerinages sur les genoux qui rappellent une fois encore une pratique largement attestée dans la Basse-Bretagne d'Ancien Régime ${ }^{27}$.

L'analyse des gwerzioù rapportant l'énonciation de testaments donne à la fois de précieuses descriptions du contexte dans lequel se déroule la dictée et des informations concernant le contenu de testaments à la longueur et à la précision parfois étonnantes. On y retrouve, depuis la formule d'introduction recommandant l'âme du défunt à Dieu et précisant le lieu d'enterrement souhaité jusqu'au détail des legs pieux, une similitude là encore souvent remarquable avec la documentation écrite : la complainte la plus singulière en la matière rapporte le testament du marquis de Guerrand décédé en 1669, dont le contenu occupe jusqu'à cinquante et un octosyllabes dans la version recueillie par Gabriel Milin ${ }^{28}$. De manière moins spectaculaire mais peut-être plus précieuse, la chanson rapporte des traces de testaments pauvres et sommaires qui se rapprochent sans doute le plus d'actes oraux dont l'écrit n'a pas gardé trace; en trois vers nous sont ainsi présentées les dernières volontés d'une jeune fille malade dont le bien-aimé est venu au chevet : "Pelec'h e man va mam, larit deï dond dam c'haouet/Ma rin va testamant breman pa oc'h deud d'em goëlet.//Dallid va alc'hoëchou, a nombr eus va habijou ${ }^{29}$."

Les indications concernant les pratiques d'enterrement sont nombreuses dans un registre de gwerzioù qui se terminent bien souvent par des morts plus ou moins édifiantes. Les bouleversements dans les usages funéraires qui apparaissent au cours de la période d'Ancien Régime permettent dans ce domaine de dater plus finement des chants ou tout au moins les interpolations tardives qui y ont été insérées.

En dehors de quelques mentions exceptionnelles d'enterrement hors de la terre consacrée, l'essentiel des précisions concernant le lieu de sépulture se rapporte à l'église ou au cimetière. La répartition entre ces deux lieux ne paraît toutefois pas coïncider avec la réalité d'Ancien Régime

27. Provost, Georges, La Fête et le sacré. Pardons et pèlerinages en Bretagne aux XVII et XVIII siècles, Paris, 1998, 530 p., p. 74-98.

28. Bulletin de la Société Académique de Brest, t. IV, 1865, p. 105-110. Les notes de Milin ayant servi à cette publication sont éditées dans le supplément "Gwerin n ${ }^{\circ} 1$ " de la revue Hor Yezh, 1961, p. 9-10.

29. " Où est ma mère, dites-lui de venir me trouver/Pour que je fasse mon testament maintenant que vous êtes venu me voir.//Prenez mes clefs et un grand nombre de mes habits. " Collection Penguern, manuscrit 90, folios 2-3. 
connue d'après les sources écrites, dans lesquelles l'enterrement dans l'église est très majoritaire malgré l'hostilité ancienne de l'institution ecclésiastique. Ce n'est réellement que suite à la déclaration royale de 1776, qui s'inscrit dans un mouvement plus général de lutte contre l'insalubrité et qui reflète une nouvelle perception de l'hygiène, que les pratiques funéraires de Basse-Bretagne s'alignent peu à peu sur les nouvelles normes instaurées à l'échelle du royaume, non sans résistance de la part des populations ${ }^{30}$. Dans la chanson, c'est pourtant essentiellement le cimetière qui est mentionné, y compris pour les nobles. C'est ainsi qu'une version de la gwerz relatant les méfaits du capitaine René de La Tremblaye du temps des guerres de la Ligue, datée des alentours de 1595, fait explicitement référence à l'enterrement au cimetière de Jeanne Le Roux, dans un contexte tout à fait ordinaire où l'on aurait attendu une sépulture à l'église ${ }^{31}$.

Une telle fréquence des inhumations au cimetière semble donc devoir être expliquée par le recours massif à des interpolations tardives venues renouveler le chant. L'analyse des différentes versions de la complainte sur le testament du marquis de Guerrand est à cet égard révélatrice : le contexte de la pièce, tant sur le plan chronologique - le décès date de 1669 - que du statut social du marquis, conduit à privilégier le choix d'une sépulture dans l'église. L'analyse de cinq versions de la gwerz où le lieu d'inhumation est précisé ${ }^{32}$ montre cependant une situation plus complexe. L'une d'entre elles mentionne un enterrement " 'bars en Iliz, pé er porched", c'est-à-dire « dans l'église ou sous le porche "; trois autres le situent " 'barz én iliz pé ér véret ", "dans l'église ou dans le cimetière "; la dernière parle uniquement du cimetière du bourg de Plouégat. Ces trois exemples révèlent pleinement les mécanismes d'interpolation d'éléments ultérieurs à la période de composition du chant. La similitude entre les expressions " er porched " et "ér véret " rend la substitution très aisée. On peut raisonnablement imaginer de même que le nom " ar vered " a pu remplacer celui de " an iliz ", "l'église ", composé du même nombre de syllabes et situé en milieu de vers, ce qui écarte le problème de la rime. Sur le plan du déroulement du récit chanté, sur le fond comme sur la forme, les modifications sont insignifiantes; sur le plan culturel, elles sont capitales ${ }^{33}$. Ces interpolations sont naturellement venues renouveler le chant au fil de la transmission orale, en recréant inconsciemment un contexte plus familier pour les chanteurs : dès lors que l'usage d'enterrer les morts à l'église a disparu

30. CROIX, Alain, op. cit., p. 1006-1028; LEMAITRE, Alain J., " Espace sacré et territoire vital au XVIII ${ }^{\mathrm{e}}$ siècle : La régulation des lieux d'inhumation en Bretagne ", Annales de Bretagne et des Pays de l'Ouest, t. 90, 1983, 2, p. 249-259.

31. Collection Penguern, manuscrit 112, folios 1-6.

32. Versions recueillies ou publiées par H. de Kerbeuzec, Anatole Le Braz, Gabriel Milin, Jean-Marie de Penguern et Madame de Saint-Prix.

33. La même analyse peut également être faite en comparant la vingtaine de versions connues de la complainte An Aotrou ar c'homt, que l'on retrouve abondamment dans le répertoire en français sous le titre de Jean Renaud, ou encore celles de la gwerz sur la mort de M. de Kerguezec datée de 1709. 
et que de nouvelles pratiques funéraires se sont imposées en BasseBretagne, cet élément du chant n'a plus été d'actualité et a été naturellement transformé. De tels apports peuvent donc même être datés : ils sont vraisemblablement postérieurs au troisième quart du XVIII ${ }^{\mathrm{e}}$ siècle.

Au contraire, une pièce comme Dom Ian ar C'haro ${ }^{34}$, dont toute la trame tourne autour de la volonté de Marie Renée d'être enterrée dans l'église malgré l'interdiction de l'évêque, pourrait être datée de façon crédible au cours de la période de tensions grandissantes qui oppose, d'une part, des autorités civiles et ecclésiastiques qui remettent en cause l'enterrement dans l'église et, d'autre part, des paroissiens pour qui la sacralisation de l'espace funèbre devient une préoccupation grandissante; cela conduirait à privilégier une datation tardive, mais antérieure à la phase de normalisation de l'enterrement au cimetière, donc vraisemblablement dans le courant du XVIII ${ }^{\mathrm{e}}$ siècle $^{35}$.

\section{Une riche palette de thèmes à aborder}

On peut dresser plus rapidement un panorama d'autres angles d'approche pour lesquels la complainte en langue bretonne s'avère d'un apport original. Sa matière peut être sollicitée dans le cadre d'une étude de la perception des gestes du respect et de l'honneur, en s'intéressant à l'analyse des comportements de rejet social ${ }^{36}$, de violence physique ou verbale qui diffèrent selon le sexe et l'origine sociale : insultes ${ }^{37}$ et atteintes à la coiffure pour les femmes, duels à l'épée pour les nobles ${ }^{38}$ et au penn-bazh - bâton à bout ferré - pour les paysans. La précision de l'arme utilisée permet de situer socialement les nombreux clercs amenés à défendre l'honneur de jeunes héritières, comme dans la gwerz concernant le clerc de Lambaul : une version mentionne celui-ci portant l'épée au côté, signe évi-

34. Collection Penguern, manuscrit 91, folios 37-38 et 58 .

35. L'absence de versions multiples pour ce chant est un indice supplémentaire en faveur d'une datation peu ancienne. Conformément à la morale de justice et d'édification véhiculée par la complainte, la gwerz donne raison aux paroissiens : quatre anges descendent du ciel et s'emparent du corps de la défunte pour l'emmener sous le porche de l'église, tandis que cinquante-trois personnes assistant à la cérémonie meurent sur place et que le recteur tombe malade.

36. Jeanne Riou refuse de croire la promesse de mariage que lui fait le seigneur de Boisriou et préfère se noyer plutôt que de rentrer chez elle déshonorée, en pensant que " p'antreinn, ar zul, en ilis,/Me 'vô diskouezet gant ar bis,//Ma larô ann eil d'egile :/-Sell serc'h 'nn aotro Koadriou aze! " : Quand j'entrerai, le dimanche, dans l'église,/Je serai montrée au doigt,//Et l'on se dira l'un à l'autre :/- Voilà la concubine du seigneur Du Boisriou! » LUZEL, Gwerziou II, p. 230-231.

37. Au sujet d'une jeune fille qui traite sa mère de " hugnodès ", c'est-à-dire de huguenote, le collecteur de cette pièce contenue dans le second volume des Soniou note que " c'est, en Bretagne, la pire des insultes ". LuzEL, Soniou II, p. 4-7. Les insultes les plus courantes sont sans doute celles ayant trait aux caquins, descendants des lépreux. Sur les caquins, voir : CroIx, Alain, "L'histoire d'un trait de mentalité. Les caquins en Bretagne ", Annales de Bretagne, t. 86, 1979/4, p. 553-564.

38. Pratique retrouvée dans plusieurs complaintes qui ont pu être précisément datées au cours de la première moitié du XvII ${ }^{\mathrm{e}}$ siècle, alors que le duel est à son apogée. 
dent de sa condition noble, tandis qu'une autre met cette fois en scène un clerc se battant au penn-bazh, donc de condition roturière.

On touche ainsi au domaine de la mise en scène des hiérarchies sociales dans la chanson, qui apparaît là encore bien plus comme le reflet de la société rurale d'Ancien Régime que de celle de l'époque des collectes. Les spécificités propres à la Basse-Bretagne relevées dans d'autres sources écrites se retrouvent transposées dans le langage de la complainte : les gwerzioù décrivent une noblesse hétérogène, oscillant entre une haute noblesse acquise au modèle de la cour royale qui étale son luxe et ses titres et une " plèbe nobiliaire ${ }^{39}$ " principalement composée de cadets appauvris dont la profusion est garantie par le système héréditaire du partage noble entériné par la Coutume de Bretagne ${ }^{40}$; cette petite noblesse pauvre rejoint et affronte, pour l'obtention des filles héritières, une élite paysanne relativement fortunée qui s'est élevée par la promotion de ses fils au rang de clercs ${ }^{41}$; à l'opposé, une profusion de pauvres bien intégrés se distingue nettement d'une catégorie d'exclus originaux notamment fournie par les caquins. Les hiérarchies socioculturelles peuvent encore se mesurer aux mentions concernant la langue utilisée par les protagonistes, dans un univers plurilingue où se côtoient breton, français et latin sans confusion selon les lieux et les milieux.

La conservation particulièrement fiable des toponymes dans la chanson, de nombreuses fois démontrée - dans la mesure où la version recueillie reste proche de l'espace dans lequel la complainte a été composée -, permet également d'envisager une étude du rapport à l'espace. L'espace vécu, entre enracinements et mobilités à différentes échelles, se décline entre géographie du quotidien et déplacements exceptionnels : la richesse des complaintes évoquant des récits de pardons et de pèlerinages, entre autres, permet d'esquisser une cartographie coïncidant avec la carte des sanctuaires effectivement fréquentés sous l'Ancien Régime ${ }^{42}$; la plupart des mentions se rapportent en effet à des pèlerinages à leur apogée dans la seconde moitié du XVII ${ }^{\mathrm{e}}$ siècle et au début du XVIII ${ }^{\mathrm{e}}$ siècle (Le Yaudet, Le Folgoët, Sainte-Anne-d'Auray) tandis que des sanctuaires à l'essor plus tardif au cours du XVIII ${ }^{\mathrm{e}}$ siècle, comme Bulat ou Sainte-Anne-la-Palud, sont presque voire totalement absents.

39. NASSIET, Michel, Noblesse et pauvreté. La petite noblesse en Bretagne, XVe-XVIII siècle, Rennes, 1993, p. 52-53.

40. Parmi beaucoup d'autres exemples, on peut citer Jeanne Le Guern qui rencontre un gentilhomme "gwisket 'vel pä̈sant ", " habillé comme un paysan ", sur la route de Guingamp. LuZEL, Gwerziou I, p. 26-27. Un proverbe populaire, qui m’a été aimablement signalé par Daniel Giraudon, rappelle une situation similaire : "Noblañs Plouha, noblañs netra" ("Noblesse de Plouha, noblesse de rien du tout ").

41. Le terme de kloareg - rendu en français par celui de clerc - qualifie cette catégorie, bien représentée dans la chanson, de fils de paysans aisés ayant poursuivi des études initialement dans le but de se destiner à la prêtrise, mais qui ne souhaitent ou n'arrivent finalement pas toujours à mener leur projet à son aboutissement. DupuY, Roger, "Chansons populaires et chouannerie ", Bulletin de la Société d'Histoire Moderne et Contemporaine, t. 25, 1978/4, p. 9-10.

42. Provost, Georges, op. cit. 
Mais l'étude peut également s'attarder sur le regard porté sur les mondes lointains, révélant un imaginaire difficile à mesurer au début du XXI ${ }^{\mathrm{e}}$ siècle : quelles images ont pu naître dans l'esprit du public de cette gwerz bien connue datant du deuxième quart du XVII ${ }^{\mathrm{e}}$ siècle qui rapporte le combat entre Les Aubrays et le "Morian goue ", le "Maure sauvage " du roi ${ }^{43}$ ? C'est une porte ouverte sur l'inconnu que constitue chaque évocation de l'Hibernie ou de la Roumanie - parfois tronquée en Mani, signe que le nom ne se réfère pas à une réalité géographique précise pour le chanteur $^{44}$-, des Islandais qui assiègent Guingamp ${ }^{45}$, de Dom Iann Derrien sur la route de "Sant Jakez an Turki ${ }^{46}$ ".

\section{La nécessaire confrontation des sources pour une mise en valeur de la spécificité des gwerzioù}

Quel que soit l'angle d'étude abordé, l'analyse culturelle des complaintes en langue bretonne ne se passe évidemment pas d'une comparaison avec d'autres sources. Cette confrontation ne s'inscrit plus nécessairement dans le but de rechercher une corrélation entre une chanson précise et un événement réel et daté, mais dans le souci de mettre en évidence la spécificité du regard porté par un milieu rural et bretonnant sur la réalité de la Basse-Bretagne d'Ancien Régime.

\section{La comparaison entre complainte en langue bretonne et canard imprimé : l'incendie de la tour de plomb de la cathédrale de Quimper en 1620}

L'originalité de la complainte, par rapport à un support écrit émanant d'un autre contexte socioculturel, apparaît nettement à travers une étude de cas privilégiée : la comparaison entre une gwerz et un imprimé datant du début du XVII ${ }^{\mathrm{e}}$ siècle ${ }^{47}$. La complainte An tour plom relate en effet l'incendie en 1620 d'une flèche de la cathédrale de Quimper recouverte de plomb : le fait divers s'articule autour de la vision d'un démon au sommet

43. LuZEL, Gwerziou II, p. 568-571. Le récit de la damnation de Katell Gollet, précipitée en enfer pour avoir refusé de confesser tous ses péchés, est pour sa part situé aux Indes, dans un cantique extrait du recueil du missionnaire Julien Maunoir au XVII ${ }^{\mathrm{e}}$ siècle (Canticou Spirituel da zisqui an hent da vont d'ar Barados, Quimper, Derrien, non daté, p. 44-48; on retrouve ce même cantique dans les éditions de Gualter Buitingh, R. Malassis ou S. Blot). La feuille volante imprimée par Lédan à Morlaix dans la seconde moitié du XIX ${ }^{\mathrm{e}}$ siècle renforce, visiblement dans un souci commercial, le caractère exotique de cette localisation; la chanson est ainsi située " er guœer a Itara, en Indes-sav-heol, er bla 1560 ", c'est-à-dire " dans la ville d'Itara, dans les Indes orientales, en l'an 1560 ». À part cette indication, le récit se déroule sans surprise dans un cadre totalement occidental et chrétien. OLLIVIER, Joseph, La chanson populaire sur feuilles volantes. Catalogue bibliographique, Quimper, 1942 , p. 156.

44. LuZEL, Gwerziou I, p. 186-193.

45. Collection Penguern, manuscrit 91, folios 51-53.

46. "Saint-Jacques de Turquie ", autre nom de Saint-Jacques-de-Compostelle.

47. La transcription des documents utilisés est donnée en annexe. 
de l'église et des moyens mis en œuvre pour le conjurer. La gwerz a pu être mise en parallèle, pour la première fois en 1864 par Gabriel Milin, avec un canard imprimé contemporain de l'événement; celui-ci, plusieurs fois réédité à Rennes et à Paris ${ }^{48}$, touche avant tout un public semi-lettré ou lettré ${ }^{49}$, urbain et francophone - tout l'inverse du public de la gwerz - friand d'une presse à sensation qui se diffuse $a u X{ }^{\mathrm{e}}{ }^{\mathrm{e}}$ siècle par l'intermédiaire de ce support. La confrontation entre ces deux types de sources, l'une en langue bretonne, versifiée et transmise oralement, et l'autre en langue française, en prose et transmise par l'écrit, est donc une confrontation entre deux cultures, deux regards différents posés sur un même événement.

\section{Les différentes versions de la complainte}

Si l'on s'attache, dans un premier temps, à confronter les six versions connues de la gwer $z^{50}$, on constate qu'elles s'appuient sur une trame similaire. On peut cependant distinguer nettement deux familles de chants : la première est composée de l'unique complainte collectée par La Villemarqué dans un rayon sans doute proche du lieu de l'événement, la seconde regroupe les cinq autres recueillies aux alentours de Morlaix. La circulation de la gwerz et de la connaissance du fait divers jusque sur les côtes septentrionales de la Basse-Bretagne n'est pas surprenante. Milin, en expliquant les circonstances dans lesquelles il a recueilli la complainte, rappelle la facilité de diffusion des nouvelles par l'intermédiaire de toute une catégorie sociale ambulante, formée de petits métiers et de mendiants, dont le répertoire de chansons est particulièrement riche : il rapporte en effet qu' " une jeune et pauvre mendiante, Perrine Poder, du Ponthou, près Morlaix, [lui] récitait, à Brest, sur la route de Paris, les vers qui vont suivre ${ }^{51}$ ». L'une des versions recueillies à Taulé substitue au nom de Quimper celui de Ploubezre, à une trentaine de kilomètres à l'est du lieu de collecte, et pourrait être liée à une réactualisation du chant autour d'un événement plus récent : en 1817, puis en 1830, le clocher de l'église de Ploubezre a été foudroyé et a dû être démoli, aucun incendie n'étant cependant évoqué ${ }^{52}$.

Des détails divergent selon les deux groupes de versions : on peut notamment relever que, dans la pièce de La Villemarqué, saint Corentin est naturellement évoqué; au nord, la mention de la Vierge lui est par contre

48. SEGUIN, Jean-Pierre, L'information en France avant le périodique. 517 canards imprimés entre 1529 et 1631, Paris, 1964, 129 p. Le même document est à nouveau publié dans le Recueil de Dissertations anciennes et nouvelles sur les apparitions, les visions et les songes, par l'abbé Lenglet-Dufresnoy, Avignon, 1751.

49. Il est vrai toutefois qu'un seul lecteur suffit pour informer par le biais de l'oral un grand nombre de ceux qui n'ont pas accès à l'écrit.

50. Extraites des collections de La Villemarqué, Luzel, Penguern et Milin. Aucun collectage oral n'a à ma connaissance été réalisé.

51. Milin, Gabriel, "La tour de plomb de Quimper ", Bulletin de la Société Archéologique de Brest, t. IV, 1864-1865, p. 90-91.

52. Couffon, R., Répertoire des églises et chapelles du diocèse de Saint-Brieuc et Tréguier, Saint-Brieuc, 1939, p. 355-356. 
préférée, signe que le nom de Corentin, premier évêque de Quimper, n'est plus évocateur. On peut de cette manière mesurer les limites géographiques au-delà desquelles le culte d'un saint, si important soit-il, n'est plus connu, tandis que la mention de la ville de Quimper résiste beaucoup mieux aux interpolations ultérieures. Le texte de La Villemarqué apporte également des éléments qui ne figurent pas dans les autres pièces, notamment des précisions temporelles ou la description du transport des objets précieux hors de l'église; les versions du nord apportent quant à elles de riches compléments, comme la description des processions autour de l'édifice, les tentatives pour conjurer le démon au moyen d'hosties, le dialogue entre un prêtre et le démon et enfin l'extinction du feu grâce à un pain de seigle consacré - ou de froment, céréale plus prestigieuse - jeté dans les flammes avec du lait de femme.

\section{La différence de regard entre gwerz et canard}

Il s'agit ensuite de confronter les textes des chansons au canard imprimé traitant également de l'incendie de la tour de plomb de Quimper. Le lien entre un canard et des complaintes en langue bretonne a déjà été relevé par Gaël Milin dans le cadre d'une série de gwerzioù mises en corrélation avec le Discours d'une Histoire et Miracle advenu en la ville de Montfort, cinq lieues pres Rennes en Bretaigne ${ }^{53}$ : dans les deux cas, un récit très similaire met en scène la même tentative de pendaison infructueuse d'une jeune fille innocente sauvée par les miracles de la Vierge; ces deux sources ne sont qu'une variation supplémentaire autour d'un thème attesté dans la culture religieuse européenne depuis le VII ${ }^{\mathrm{e}}$ siècle. Le cas de l'incendie de Quimper est très différent, puisque l'événement n'a pas d'antécédent et ne s'inspire visiblement d'aucun modèle antérieur. La différence entre la gwerz et le canard, tant dans le traitement du sujet que dans les publics concernés, rend peu probable une influence réciproque. Le caractère exceptionnel et brutal de la destruction de la cathédrale a dû inspirer parallèlement ces deux sources indépendamment l'une de l'autre.

Le canard se doit d'être attractif, et utilise pour cela des procédés que l'on retrouve dans les chansons sur feuilles volantes qui voient l'apogée de leur diffusion en breton au XIX ${ }^{\mathrm{e}}$ siècle et qui constituent une des évolutions de cette presse à sensation. La longueur et le style emphatique du titre sont le premier point d'accroche pour le public; l'intitulé se plie ici parfaitement aux canons du genre et utilise un lexique mélodramatique de circonstance, abondamment employé tout au long de la narration. Les descriptions sont plus complètes dans l'imprimé (comprenant entre autres des détails sur la tour ou sur le démon), là où la concision exigée par la versification empêche de longs développements dans la chanson.

53. MııIN, Gaël, « De Saint-Jacques-de-Compostelle à Notre-Dame-du-Folgoët : les voies de l'acculturation ", Annales de Bretagne et des Pays de l'Ouest, 1994, t. 101, 3, p. 7-47. 
Le choix des précisions temporelles indique deux conceptions du temps très différentes. L'une, urbaine, rythmée par la sonnerie régulière de l'horloge, est donnée en valeurs chiffrées, qui continuent avec l'estimation monétaire des dégâts provoqués par l'incendie. Dans les complaintes, les précisions de cet ordre sont quasi inexistantes, si ce n'est l'indication temporelle de la nuit de Noël au cours de laquelle a eu lieu la profanation de la cathédrale ou encore, dans la version de La Villemarqué, la datation de l'incendie " da gouel Sant gregor mintat ${ }^{54}$ ". On sent bien le peu de pertinence de telles informations pour l'auditoire des gwerzioù, pour lequel la mention du calendrier liturgique est beaucoup plus évocatrice en ce début du $\mathrm{XVII}^{\mathrm{e}}$ siècle. Ce constat permet de mieux comprendre le peu de fiabilité des dates relevées dans les complaintes et l'habituelle absence complète de données temporelles chiffrées, hormis dans les gwerzioù les plus récentes, déjà imprégnées par une notion mathématique du temps.

Quel que soit le support utilisé, le sens de la vue occupe une place primordiale, l'univers sonore étant également évoqué (cloches, chants des processions, voire musiciens) : dans une société où l'écrit est encore très minoritaire, la vue est une preuve de véracité, la vision collective étant encore un degré supplémentaire dans la recherche d'une garantie dans les propos.

L'accent est mis sur l'encadrement religieux, le nombre et le rôle des ecclésiastiques dans l'extinction de l'incendie. Le récit du canard se termine en apothéose par la bénédiction d'un pain de seigle et d'une hostie jetés dans le feu, qui mettent fin à l'incendie en renvoyant le démon. Cette mise en scène édifiante, qui place l'intervention du clergé au premier plan par un acte qui a trait au miracle, diffère cette fois nettement de la relation de l'événement dans les gwerzioù. Toutes les versions font intervenir, en plus du pain de seigle, dont le caractère sacré n'est mentionné que dans une pièce, le lait de la poitrine d'une femme de dix-huit ans en train d'allaiter son premier enfant. C'est même l'unique remède à l'incendie dans la pièce de La Villemarqué ${ }^{55}$. Dans une autre version, le moyen de conjurer le démon n'est plus donné par le clergé mais par "ar brofeded ", " les prophètes ". Le poids de l'encadrement religieux apparaît donc de manière nettement plus sensible dans le canard dont le but d'édification est clair, là où la gwerz, par sa diffusion orale, est moins facilement contrôlable par l'Église et révèle des pratiques religieuses peu orthodoxes en comparaison des nouvelles exigences et de l'esprit de la Réforme Catholique.

54. "À la Saint Grégoire de bon matin. "

55. Gwennolé Le Menn remarque que l'utilisation du lait de femme pour combattre le feu allumé par un démon ne semble pas attestée par ailleurs dans la tradition populaire bretonne. Par contre, deux cantiques extraits des Canticou spirituel du père Maunoir font de cette faculté un privilège réservé à la Vierge, notamment un Pater en enor dan diuuron sacr ar Verc'hes ("Pater en l'honneur des seins sacrés de la Vierge "); la question d'une récupération par l'Église de pratiques antérieures peut alors être posée. LE MENN, Gwennolé, La femme au sein d'or, Saint-Brieuc, 1985, p. 119-122. 
Malgré ces deux contextes extrêmement différents, la similitude dans le choix des épisodes relatés est frappante : les deux publics touchés par ces récits semblent demandeurs des mêmes informations; c'est le traitement de celles-ci qui diffère.

\section{L'évolution de la perception de l'événement}

Au-delà de la comparaison entre ces deux supports, d'autres sources contemporaines et postérieures à l'incendie permettent de s'attarder sur la manière dont la perception de l'événement s'est modifiée au cours des siècles.

L'incendie de la cathédrale de Quimper est en effet connu également par la conservation d'une délibération capitulaire prise le 7 février 1620, ayant pour objet " les réparations et restauration des ruines nouvellement arrivées par le tonerre et feu à [l']église cathédrale " et les indemnisations financières à distribuer " entre les ouvriers blécés, et qui se seroint exposés au péril et danger de leur vie, pour estaindre le feu et garantir ladite église $^{56}$ ". Le document ne fait aucune mention des circonstances exceptionnelles du fait divers. Ce n'est certes pas l'objet de la délibération, mais il est notable que seul le tonnerre est donné comme cause de l'incendie. On se trouve ici en présence d'un autre public que ceux des gwerzioù ou des canards : dans un univers encore résolument tourné vers une explication magique du monde, ces derniers ne remettent jamais en doute l'apparition du démon, qui est tout aussi rationnelle que la cause de l'incendie et le miracle de son extinction par un pain bénit ou par du lait de femme.

L'événement n'est pas regardé du même œil, seize ans plus tard, par le théologien dominicain Albert le Grand, qui cherche de plus amples informations sur les circonstances de l'épisode. De son enquête n'a été retrouvée qu'une lettre demandant des précisions sur la cause de l'incendie ${ }^{57}$, ainsi que la conclusion tirée de ses recherches, reprise dans le Dictionnaire géographique et historique d'Ogée en ces termes : "1620. - L'aiguille de plomb au centre de la croisée de la cathédrale est, dit Albert le Grand, fondue par un étrange accident ${ }^{58}$. " L'événement est lu dans ce courrier avec un regard critique, et le théologien préfère passer sous silence les circonstances extraordinaires de la catastrophe; malgré l'incertitude qui subsiste autour de cette description laconique et du sens exact entendu par Albert le Grand lors de la rédaction de cette note, il semble bien qu'un nou-

56. Texte cité dans LE MEN, René-François, Monographie de la Cathédrale de Quimper, Quimper, 1877, p. 218-222. La transcription complète de toutes les sources utilisées au cours de ce développement est disponible sur le site [www.chez.com/follenn/tourplomb] grâce au travail réalisé par Thierry Rouaud et Serge Nicolas.

57. "Lettre du R. P. Albert le Grand ", Revue de Bretagne et de Vendée, 1857/2, p. 424426. La lettre, datée de 1636 et adressée au marquis de Rosmadec, est publiée par Arthur de La Borderie.

58. Oget, Jean, Dictionnaire historique et géographique de la province de Bretagne, Rennes, 1853 (rééd.), 2e vol., p. 425. 
veau mode de raisonnement s'affirme parallèlement à l'explication magique fournie par la chanson et le canard.

René-François Le Men, deux siècles et demi plus tard, relate le récit de l'incendie, évidemment à la lumière d'une explication scientifique désormais incontestée, et rappelle comment " l'imagination populaire, fortement frappée, crut voir des phénomènes surnaturels ${ }^{59}$ ". Avant lui, Jean-François Brousmiche rapporte encore cette histoire, de même que l'existence du canard qu'il dit avoir lu, parmi les nombreuses anecdotes qui parsèment ses notes de voyage dans le Finistère ${ }^{60}$. Les circonstances de l'épisode sont désormais passées complètement dans le domaine de la fiction, du merveilleux. Elles fournissent d'ailleurs le thème d'un récit fantastique écrit par Tanguy Malmanche au début du xx ${ }^{\mathrm{e}}$ siècle, intitulé La Tour de Plomb ${ }^{61}$. Le même sujet est repris par Maryvonne Méheut en 1944 dans une peinture destinée à illustrer une édition des Veillées Bretonnes de Luzel ${ }^{62}$.

Un élément important manque à cette énumération de regards portés sur l'événement : les commentaires des chanteurs, dont on ne garde aucune trace. L'évolution de la perception de l'épisode n'est donc possible que du côté du public lettré et francophone. La remarquable similitude entre les éléments rapportés dans le canard et ceux rapportés dans les gwerzioù donne à penser que le chant initial s'est comme souvent très bien conservé. On ne doit pas conclure pour autant à une indéfectible conservation d'une culture rurale et bretonnante, mais plutôt à une remarquable fiabilité dans la transmission de la chanson au cours du temps.

\section{L'intérêt d'une analyse comparée entre complaintes et archives judiciaires d'Ancien Régime}

La comparaison entre complainte et imprimé est l'exemple même du document exceptionnel dont l'analyse ne peut être généralisée faute de matériaux actuellement accessibles. Si ce n'est l'étude déjà évoquée de Gaël Milin, aucun autre document de cette nature n'a pu être mis en relation avec une gwerz, d'où la nécessité de se tourner vers d'autres supports. Le recours aux archives judiciaires d'Ancien Régime pourrait laisser penser, dans un premier temps, qu'un semblable écueil sera vite rencontré : les tentatives de concordance entre une chanson précise et un événement ayant donné lieu à procès ont montré à la fois la richesse des résultats obtenus dans quelques beaux cas d'études - notamment la remarquable analyse de Donatien Laurent sur la gwerz de Louis Le Ravallec ${ }^{63}$ ou encore le procès du sieur de Penanger étudié par Michel Nassiet puis Daniel

59. Le Men, Gwénolé, op. cit., p. 219.

60. Brousmiche, J.-F., Voyage dans le Finistère en 1829, 1830 et 1831, Quimper, 1977, t.2, p. $265-266$.

61. Malmanche, Tanguy, Contes, 1975, p. 115-179.

62. LUZEL, F.-M., Veillées Bretonnes, Paris, 1944, page de garde.

63. LAUREnT, Donatien, " La gwerz de Louis Le Ravallec", Arts et Traditions Populaires, 1967, 1, p. 19-79. 
Giraudon ${ }^{64}$ - mais également les limites d'une méthode qui ne permet qu'un traitement de cas exceptionnels.

Pourtant, une autre approche comparative entre ces deux sources semble envisageable dès lors que l'on s'intéresse non plus à la mise en évidence d'une datation précise de nouvelles complaintes, mais à l'analyse des données culturelles qu'on relève dans celles-ci. Les archives criminelles apparaissent alors comme une source privilégiée de comparaison par le nombre des similitudes qu'elles présentent avec les gwerzioù.

Les faits qui y sont relatés se rapprochent tout d'abord grandement de ceux rencontrés dans les complaintes, dans lesquelles se côtoient des crimes de toute nature qui sont la plupart du temps la raison même de la composition du chant. En outre, les procédures criminelles tout comme les chansons en langue bretonne - et c'est là ce qui nous intéresse le plus -, foisonnent de détails se rapportant à la vie quotidienne, aux comportements et aux sensibilités; qui plus est, les milieux les moins favorisés y sont massivement représentés, chose précieuse dans le domaine de l'archive écrite. Les deux sources sont animées par le même souci de vraisemblance, de crédibilité dans le discours qu'elles proposent, ce qui assure une grande fiabilité aux informations données : les gwerzioù se caractérisent par le caractère vrai - ou censé l'être - de l'histoire qu'elles rapportent, transmettre une information erronée revenant à remettre en cause la considération et le respect dus au chanteur; les accusés ou les témoins doivent quant à eux élaborer un discours basé sur des faits vraisemblables, à défaut d'être vrais, pour convaincre les juges de leur bonne foi et de leur innocence. Il est frappant de voir comme la définition de cette " règle impérative de vraisemblance ${ }^{65}$ " concernant les archives judiciaires est conforme à celle qui caractérise les complaintes : « Non seulement leur contenu exhibe un monde organisé (ou démantelé), mais leur énonciation est là pour provoquer de la conviction et entraîner l'assentiment de ceux qui écoutent et qui jugent. Dans le rapport étroit entre la parole dite et la volonté de créer du vraisemblable s'instaure l'événement. " Précisons là qu'il s'agit de l'analyse d'Arlette Farge ${ }^{66}$ concernant les archives judiciaires parisiennes, tant il est vrai qu'une réflexion identique aurait pu être formulée dans un contexte tout autre par Donatien Laurent au sujet d'un auditoire bretonnant qui, lui aussi, écoute et juge.

De fait, la similitude entre les faits relatés dans les gwerzioù et dans certaines procédures criminelles est remarquable : au-delà de cas exceptionnels comme l'affaire Le Ravallec pour laquelle une procédure criminelle étoffée correspond précisément au meurtre relaté dans la chanson ${ }^{67}$, la

64. NASSIET, Michel, La France du second XVII siècle. 1661-1715, Paris, 1997, p. 109-111; GiRAudon, Daniel, " Penanger et de La Lande, Gwerz tragique au XVII ${ }^{\mathrm{e}}$ siècle en Trégor ", Annales deBretagne et des Pays de l'Ouest, t. 112, n 4, 2005, p. 7-42.

65. MuchemblED, Robert, La Violence au village. Sociabilité et comportements populaires en Artois du XV eu XVII siècle, Turnhout, 1989, p. 18.

66. FARGE, Arlette, Le goût de l'archive, Paris, 1997, p. 99.

67. Arch. dép. du Morbihan, B 2944. 
relation du procès de François Le Tanaf, qui égorge pour la dévaliser Jeanne Cadic qu'il a rencontrée sur un chemin des alentours de Tréguier ${ }^{68}$, ne laisse pas indifférent lorsqu'on a à l'esprit la complainte de Mauricette Tefetaou assassinée par un marchand de fil alors qu'elle gardait ses moutons ${ }^{69}$; le viol de Jeanne Rolland, âgée de quinze ans, par deux écuyers entrés chez elle en lui faisant croire qu'ils venaient chercher du lait, dans une affaire portée en justice en $1680^{70}$, n'est pas sans rappeler la gwerz de Marie Le Masson déshonorée par un baron puis rejetée par sa famille ${ }^{71}$; l'accusation d'infanticide portée contre Isabelle Riou, domestique chez le baron de Beaulieu en Trégor et enceinte des œuvres de son maître ${ }^{72}$, ou l'affaire portant sur la découverte d'un nouveau-né retrouvé mort étranglé dans la terre ${ }^{73}$, se font l'écho de la multitude de chansons rapportant de tels récits. Le constat serait d'ailleurs identique si l'on prenait en exemple des procédures extérieures à la Basse-Bretagne.

Cependant, au-delà de ces premières et partielles ressemblances concernant la nature de l'acte qui justifie la mise en chanson ou le procès, c'est surtout dans la description de comportements et de réalités matérielles mentionnés au détour d'un vers ou d'une argumentation que la similitude entre les deux sources est flagrante et que se révèle la véritable richesse de cette documentation pour une recherche en histoire culturelle : l'évocation d'un charpentier, près de Mende, "vêtu d'une veste de couleur brune à la manière des gens de Saugues ${ }^{74}$ ", de rondes à Saint-Omer en 1608 dans lesquelles " pour aller au milieu failloit avoir barbe ${ }^{75}$ " ou des leçons d'écriture données par le clerc du village de Vimy en Artois à des jeunes gens après leur journée de travail ${ }^{76}$ sont autant de précieux détails fournis dans les archives judiciaires que l'on pourrait tout aussi bien retrouver dans les chansons en langue bretonne.

68. Arch. dép. des Côtes-d'Armor, B 3567. Affaire rapportée dans HERvE, Agnès, Sur les pas de mes ancêtres... Culture, sensibilités et comportements en Trégor d'après les archives judiciaires de Tréguier, Plouguiel-Plougrescant et La Roche-Derrien (1702-1790), mémoire de maîtrise d'histoire, Rennes, 2002, non publié, volume d'annexes p. 25.

69. LuZEL, Gwerziou II, op. cit., p. 288-291.

70. Arch. dép. d'Ille-et-Vilaine, 1Bn 646. Affaire citée dans LEMornE, Sébastien, Sociabilité, comportements et mentalités nobiliaires de 1650 à 1750 d'après les archives de la cour d'appel de la chambre de la Tournelle du Parlement de Bretagne, mémoire de maîtrise d'histoire, Rennes 2, 1998, non publié, p. V-VI.

71. LAURENT, D., 1989, op. cit., p. 95-97.

72. Arch. dép. d'Ille-et-Vilaine, 1 Bn 692. Affaire citée dans BuRTin, Adeline, « Je seré mariée avant que soit trois jours chantés ». Culture, sensibilité et comportements des femmes à travers les archives de la Tournelle du Parlement de Bretagne (1600-1689), mémoire de maîtrise d'histoire, Rennes 2, 1998, non publié, p. 22.

73. Arch. dép. des Côtes-d'Armor, B 3564. Herve, A., op. cit., p. 9.

74. CASTAN, Yves, "Mentalités rurale et urbaine à la fin de l'Ancien Régime dans le ressort du Parlement de Toulouse d'après les sacs à procès criminels. 1730-1790 ", ABBIATECI, A. et BILlacols, F., Crimes et criminalités en France XVII -XVIII siècles, Cahier des Annales, $\mathrm{n}^{\circ}$ 33, Paris, 1971, p. 171.

75. Muchembled, R., op. cit., p. 169.

76. Ibidem, p. 381. 
Pourtant, si la nature des données offre de nombreuses similitudes, il existe une différence notable dans la manière dont l'information est relatée et transmise : dans les procédures criminelles, il est fait recours à un écrivant extérieur, d'un milieu socioculturel autre que la grande majorité des hommes et des femmes qui comparaissent, traduisant à la fois la langue de l'oral dans celle de l'écrit et passant du breton au français par l'intermédiaire d'un traducteur explicitement mentionné; dans la complainte au contraire, cette double traduction n'est pas opérée ${ }^{77}$. De plus, d'autres enjeux que ceux de la vraisemblance doivent être pris en considération si l'on veut comprendre l'inadéquation, sur certains points, entre les faits relatés dans ces deux sources, par exemple la récurrence des mentions à l'auberge dans les archives écrites et sa quasi-inexistence dans les complaintes, ou au contraire le nombre assez élevé de gwerzioù concernant des affaires de viols ou d'infanticides comparées aux plus rares affaires criminelles s'y rapportant : audelà de ces deux exemples, c'est tout un questionnement autour du regard porté sur la société bretonnante d'Ancien Régime qu'il s'agit d'élaborer en tenant compte d'une sensibilité différente selon les sources.

\section{Conclusion : les perspectives d'exploitation des complaintes en langue bretonne dans le cadre d'une analyse en histoire culturelle}

Il apparaît donc bien que, malgré les incertitudes chronologiques qui incitent à toujours se situer dans la sphère de la vraisemblance bien plus que dans celle de la "vérité ", la mise en évidence dans les gwerzioù de faits, de comportements et de sensibilités se rapportant à la Bretagne d'Ancien Régime peut être esquissée dans la perspective d'une constante confrontation avec des sources écrites précisément datées. Parallèlement à la démonstration depuis le $\mathrm{XIX}^{\mathrm{e}}$ siècle de l'apport des complaintes en tant que source pour l'histoire événementielle, cette démarche d'analyse permet de faire apparaître un univers culturel largement en adéquation avec celui que donnent à voir les sources écrites de l'époque d'Ancien Régime.

L'exploitation des chansons populaires en langue bretonne en tant que source pour l'histoire s'avère ainsi extrêmement prometteuse : la documentation est foisonnante, elle se compte facilement en milliers de pièces mal répertoriées pour les collectes écrites et est encore plus fournie pour des collectes orales qui continuent d'être alimentées, même si les enregistrements récents sont de moins en moins nombreux et réalisés hors contexte. Dans le cadre d'une étude sur la période d'Ancien Régime, elle constitue un apport exceptionnel : à côté des sources écrites extrêmement

77. Les collecteurs constituent certes des écrivants extérieurs, mais qui ne sont censés effectuer qu'un travail de copie et non de réécriture (cette règle aujourd'hui impérative dans toute enquête orale est toutefois inégalement suivie au XIX ${ }^{\mathrm{e}}$ siècle, les critères de scientificité répondant alors à des exigences différentes de celles qui sont actuellement admises). Ce filtre disparaît en revanche totalement dans le cadre des enregistrements de chanteurs au $\mathrm{Xx}^{\mathrm{e}}$ siècle. 
abondantes qui émanent quasi exclusivement d'un milieu urbain, lettré et francophone, la chanson apporte un regard radicalement opposé, qu'aucune autre source ne permet d'appréhender avec une telle richesse : c'est, à travers le prisme déformant de la gwerz, le regard d'une société rurale, largement non-lettrée et bretonnante.

La comparaison entre sources orales et sources écrites, tout en garantissant la fiabilité de l'information relevée dans la chanson, doit permettre la mise en valeur de la spécificité du répertoire chanté. Par la confrontation entre complaintes en langue bretonne et procédures criminelles notamment, entre deux ensembles documentaires qui, au-delà d'évidentes différences formelles, présentent également un certain nombre de similitudes quant à leur contenu, c'est la mesure de deux sensibilités différentes que l'on cherche à saisir ou tout au moins à entrevoir, la compréhension de deux regards distincts et complémentaires posés sur la société rurale et bretonnante d'Ancien Régime ${ }^{78}$.

78. Je tiens à remercier Robert Bouthillier, Daniel Giraudon, Philippe Hamon, Donatien Laurent et Florent Quellier pour leurs remarques et relectures attentives. 


\section{Annexe - Complainte et canard au sujet de l'incendie de la tour de plomb de la cathédrale de Quimper en 1620}

\section{An tour plom}

Kenta vellas an tan en tour plom

Eur vugel divar brec'h e vam.

Hag e lavaras da Plouberis

E man krog an tan en ho c'hillis.

Siwas er c'hreis e man ive.

Kris a viche ar galon na voelche

En goerret plouber an eb a vize

E vellet tri person a tregont

Ebars ar verret oc'h e neum respont.

Da gout na pini vize an ardissa

Da pignat var an tour da genta.

Person Plouber a lavare

Ebars an tour dre ma pigne :

Me non ket evid mond aman

Gant ar plom bervet o diveran

Dre ma koue leski a ra.

E man an erouant var beg an tour Hag en henon evel eur skour.

- Petra klaskez var dro va zi

Me non ket bet en ta hini.

- Ta illis a zo interdisset

Gant eur plac'h iaouank hag eur kloarek

O pec'hi heni noz nedellek.

Kenta lazas an tan en tour plom Voa bara segal a lez bron.

\section{Le clocher de plomb}

Le premier qui vit le feu dans le clocher de plomb

Fut un enfant dans les bras de sa mère.

Et il dit aux habitants de Ploubezre :

"Le feu prend dans votre église. "

Hélas il est aussi au milieu.

Cruel eût été le cœur de celui qui n'eût pleuré

Dans le cimetière de Ploubezre, s'il y avait été,

En voyant trente-trois prêtres

Se répondant les uns aux autres dans le cimetière,

Pour savoir qui serait le plus hardi

Pour monter sur la tour en premier.

Le curé de Ploubezre disait

Dans la tour, à mesure qu'il montait :

«Moi je ne peux pas aller ici

Avec le plomb bouillant qui coule;

En coulant il brûle."

Le démon est sur le sommet du clocher Et il est là comme un milan.

"Que cherches-tu autour de ma maison, Moi je ne suis pas allé dans la tienne?

- Ton église est profanée

Par une jeune fille et un clerc

Qui y ont péché la nuit de Noël. »

La première chose qui éteignit le feu

Fut du pain seigle et du lait d'un sein de femme, 
Lez a peultrin eur wreg trivarc'h bla

E vaga e bugel kenta.

Kris vije ar galon na voëlche

En goeret Plomber neb a vije,

E voëlet ar sent ag ar sentezet

Digasset tout da gorn ar verret.

O vellet a Verc'hes Vari

Renket diloja deus e zi.
Le lait de la poitrine d'une femme de dixhuit ans

En train d'allaiter son premier enfant.

Cruel eût été le cœur de celui qui n'eût pleuré

Dans le cimetière de Ploubezre, s'il y avait été,

En voyant les saints et les saintes

Amenés tous au coin du cimetière,

En voyant la Vierge Marie

Forcée de sortir de sa maison.

Collection Penguern, manuscrit 112, Bibliothèque Nationale de France, folios 203-204, publié dans : Dastumad Pennwern, "Gwerin n 5 ", Hor Yezh, 1963, p. 167-168. Traduction littérale : E. Guillorel. La gwerz a été recueillie par Penguern en 1851 à Taulé près de Morlaix auprès de Jannet Kerguiduff, fileuse de laine.

Le Grand Feu, tonnerre et foudre du Ciel, advenus sur l'Eglise cathedralle de Quimpercorentin en basse Bretainne : ensemble la vision publique d'un horrible \& tres-espouvantable Demon sur ladite Eglise dans ledit feu, le premier jour de Fevrier 1620

Samedy premier jour de Fevrier mil six cens vingt advint un grand malheur et desastre en la ville de quimpercorentin c'est une belle et haute Pyramide couverte de plomb estant sur la nef de la grande Eglise et sur la croisee de la dite nef, fut toute brulee par la foudre et le feu du Ciel, depuis le haut jusques à ladite nef, sans pouvoir y apporter aucun remede. Et pour sçavoir le commencement et la fin, c'est que ledit jour sur les sept heures tendant à huict du matin, se fit un coup de tonnerre et éclairs terribles entre autres : et à l'instant fut visiblement veu un demon horrible et espouvantable, en faveur d'une grande onde de gresle se saisir de ladite Pyramide par le haut et au-dessous de la Croix, estant ledit demon, de couleur verte, ayant une longue queuë de pareille couleur. Aucun feu ny fumée n'apparut sur ladite pyramide qu'il ne fust pres d'une heure apres midy, que la fumee commença à sortir du haut d'icelle, et dura fumant un quart d'heure; et du mesme endroict commença le feu à paroistre peu à peu en augmentant tousjours ainsi que il devaloit de haut en bas : tellement qu'il se fit si grand et si espouvantable que l'on craignoit que toute l'Eglise fust brulee, et non seulement 
l'Eglise, mais aussi toute la ville tous les tresors de ladite Eglise furent tirez hors : les voisins d'icelle faisoient transporter leurs biens le plus loing que ils pouvoient de peur du feu. Il y avoit plus de quatre cens hommes pour devoir tuer le feu et n'y pouvoient rien faire. Les Processions allerent à l'entour de l'Eglise et aux autre Eglises chacun en priere. Enfin ce feu alloit tousjours en augmentant ainsi qu'il trouva plus de bois. Finalement pour toute resolution on eu recours à faire mettre des Reliques sainctes sur la nef de ladite Eglise pres et au devant du feu. Messieurs du Chapitre commencerent à conjurer ce meschant Demon, que chacun voyoit apertement dans le feu, tantost vert tantost jaune tantost bleu, jetant des Agnus Dei dans iceluy, et plus de cent cinquante barriques d'eau, quarante ou cinquante chartees de fumier, et neantmois le feu continuoit. Et pour derniere resolution l'on fit jeter un pain de seigle de quatre sols, dans lequel on y mit une Hostie consacrée puis on print de l'eau beniste et la jeta on dans le feu; tout aussi tost le Demon fut contrainct de quitter le feu; et avant que de sortir il fit un si grand remu-mesnage, que l'on sembloit estre tous bruslex, et qu'il devoit emporter l'Eglise et tout avec luy : Et en sifflant il sortit a six heures et demie du soir dudit jour, sans faire autre mal (Dieu mercy) que la totale ruine de ladite Pyramide, qui est de consequence de douze mille escus du moins. Ce meschant estant hors, on eut raison du feu. Et peu de temps apres, ledit pain de seigle se trouva encore en la mesme essence qu'il estoit, sans estre aucunement endommagé, fort que la croute estoit un peu noire. Et sur les sept ou huict heures et demie apres que tout le feu fus esteint, la cloche sonna pour amasser le peuple, afin de rendre graces à Dieu. Messieurs du Chapitre avec les Choristes et Musiciens chanterent le Te Deum et un Stabat mater dans la Chapelle de la Trinité, à neuf heures du soir. Graces à Dieu il n'est mort personne, fors trois ou quatre blessez. Il n'est pas possible de voir chose plus horrible et plus espouvantable qu'estoit ledit feu.

Rennes, J. Durand, 1620, 8 feuillets, Bibliothèque Nationale de France. Transcription : Thierry Rouaud. 


\section{RESUME}

En quoi une lecture des complaintes en langue bretonne sous l'angle de l'histoire culturelle permet-elle l'analyse d'une source orale envisagée dans sa globalité et non plus seulement par le biais de quelques-unes de ses pièces les plus remarquables? À travers le prisme déformant de la chanson, qui répond à des canons esthétiques et moraux, et sans négliger une nécessaire confrontation avec d'autres sources écrites, l'enjeu est de cerner le regard que porte une société rurale et bretonnante sur elle-même dans la BasseBretagne d'Ancien Régime.

\section{ABSTRACT}

We can study old Breton ballads as a document for cultural history, considering this oral material in a global way and not only as a collection of beautiful pieces. We know that the songs are not a faithful mirror of reality: they observe esthetic and moral rules, so that historians have necessarily to compare them with written documents. However, Breton ballads seem to be a very unusual and precious material to understand how a rural and Breton-speaking society talks about itself between the $16^{\text {th }}$ and the $18^{\text {th }}$ Century. 